\title{
Uplink Channel Assignment in Cognitive Radio WMNs Using Physical Layer Network Coding
}

\author{
Sharhabeel H. Alnabelsi, Ahmed E. Kamal, and Tasneem H. Jawadwala \\ Dept. of Electrical and Computer Eng., Iowa State University, Ames, IA, USA 50011 \\ E-mail:\{alnabsh,kamal,tasneemj\}@iastate.edu
}

\begin{abstract}
In this paper, we introduce a low overhead scheme for the uplink channel allocation within a single cell of Cognitive Radio Wireless Mesh Network (CR-WMNs). The scheme does not rely on using a Common Control Channel (CCC). The mechanism is based on Physical layer Network Coding $(P N C)$, in which two Secondary Users (SUs) are allowed to transmit synchronously over a randomly selected channel from a set of available channels, and without coordination for the purpose of requesting channels. The Mesh Router (MR) can detect up to 2 requests on the same channel due to the use of $P N C$, and replies back with a control packet which contains information about the assigned channel.

We propose two $P N C$ modulation schemes, $P N C_{1}$ and $P \mathrm{NC}_{2}$, where initially SUs choose one of them to employ through the network operation. Decoding the received signals in $P N C_{1}$ and $P N C_{2}$ depend on their received energy and phases shifts, respectively. Simulation results show that the proposed mechanism significantly outperforms traditional schemes that rely on using one CCC, or do not use $P N C$ in terms of channel allocation time.
\end{abstract}

\section{INTRODUCTION}

Due to the temporal and spatial underutilization of licensed spectrum bands, as well as crowdedness of unlicensed bands, a new spectrum access paradigm has been recently proposed namely, Cognitive Radio (CR) [1]. CR enables users to adjust their transceivers frequencies depending on the available frequency bands [2], [3]. Thus unlicensed wireless users, called SUs can dynamically and opportunistically access unused licensed bands to increase their throughput and service reliability such that whenever the licensed or the Primary Users (PUs) become active, SUs vacate their bands.

Although CR networking is a promising technique, it has many challenges such as spectrum sensing, management, mobility, allocation and sharing [4], [5]. Consequently, channel allocation and MAC protocol design become more challenging due to sporadic channel availability, lack of SUs cooperation, or hardware limitations. Thus, to achieve coordination between transmitters and receivers, a single common control channel [6], a set of common channels to all SUs [7], or multiple local control channels for SUs groups [8] have been proposed as solutions. In [10] a new MAC protocol for multi-hop Cognitive Radio Networks (CRNs) is developed to avoid using a CCC by dividing the total time into a set of intervals where each interval represents one of the available channels. In [11] a swarm intelligence method is proposed to dynamically find

This research was supported in part by the National Science Foundation under grants CNS-0626822, ECS-0601570, and ECCS-0926029. and manage control channels, since the CCC may be unknown at the first deployment time. Also, fixed CCC will increase the cost and vulnerability especially in licensed spectrum bands. Recently, the use of network coding [9] was proposed in order to allow the exchange of control information robustly and expeditiously in [13]. In this paper, we propose to use physical layer Network coding, which allows multiple packets to combine in air through interference [12], for requesting channel assignment by multiple users.

\section{Motivation}

A CR network usually employs a CCC that is used to allow exchange of control information among users in the network. This causes a wastage in network resources when there is no control data to exchange between SUs. Also, CCC can be a source of delay, especially if SUs do not listen to the same CCC simultaneously. This motivated us to try to develop a coordination strategy between SUs in a CR-WMN which includes the clients in WMN and the MR without using a CCC. We propose to employ $P N C$ in order to reduce the channel allocation overhead by the MR.

In our scheme, SUs which act as mesh clients are allowed to combine their transmissions over the same channel using $P N C$. We use this strategy for requesting uplink channels from the MR. If one or two SUs send channel requests on the same channel, $P N C$ allows the MR to extract such requests, hence increasing the probability of success $\left(p_{s}\right)$ for request transmissions. However, $P N C$ requires a strict synchronization between transmissions of SUs to MR.

We propose two $P N C$ schemes $P N C_{1}$ and $P N C_{2}$ where decoding the received signals in $P N C_{1}$ and $P N C_{2}$ depends on their received energy and phases shifts, respectively. The performance metrics values for the these two modulation schemes are different in terms of the $p_{s}$ for transmission to MR and the network setup time overhead to allocate channels to SUs by MR. For instance, due to a high environment noise, it may be better to choose $P N C_{2}$ scheme because its decoding operation depends on the phase shifts rather than energy of the received signals. Our contribution in this paper is the introduction of a channel request strategy that does not require a CCC. The strategy results in a reduced time overhead for channels allocation to client SUs.

The rest of this paper is organized as follows. The system model is explained in Section III. In Section IV, we present the physical layer network coding model which is employed in 
our proposed strategy. Section $\mathrm{V}$ describes the communication protocol between SUs and the MR in the uplink direction. Section VI shows the results, evaluation and discussion of the proposed protocol through simulation. Finally, we conclude the paper in Section VII.

\section{System ModeL}

A WMN consists of a set of clusters where each cluster is managed by an MR. Each of the clients and the MR in the cluster are within transmission range of each other. We concentrate on uplink (from client SUs to MR) channel allocation. Let the number of client SUs in a cluster and the available channels at the MR be M and N, respectively. The assumptions as follows:

- The MR has $\mathrm{N}$ transceivers for its $\mathrm{N}$ available channels. ${ }^{1}$

- SUs have fixed locations in WMN cluster and each SU has one transceiver.

- There is no coordination among SUs while selecting or accessing channels. This means that an SU does not know if it is transmitting on a certain channel simultaneously with other SUs.

- The proposed model is receiver based where the receiver is the MR in the uplink direction from the SUs in the WMN cluster.

- The SUs' transmissions are perfectly synchronized which is a requirement of $P N C$. The transmission time, frequency and phase are synchronized. Simple and effective synchronization techniques for a group of transmitters to a receiver have been proposed in [14], [15], [16]. Those techniques are used periodically by SUs to ensure synchronization in a WMN cluster and to adjust any time, frequency or phase drifts. Also, assume the environment is noise free.

- Transmission power management is used, where SUs transmission power levels are adjusted such that signals received by the MR from the different SUs have the same or very close power levels.

\section{Physical layer Network Coding Model:}

Physical layer Network Coding $(P N C)$ is utilized in packet recovery to allow multiple access over the same channel and at the same time. $P N C$ has been applied to the physical layer to increase the network capacity [12]. $P N C$ is different from Digital Network Coding (DNC) where the coding arithmetic is applied on the digital bit streams when they are received. However, in $P N C$ concurrent and synchronously received electromagnetic waves are utilized in coding operation.

The goal of our protocol is to reduce the CRN setup time which refers to the overhead time required to assign the available channels at the MR to SUs. A complete setup communication protocol is described later in Section V. In our proposed protocol, $P N C$ is used only for network setup where the control packets are too short and only contain the SU's ID to enable the MR to assign channels properly to SUs.

\footnotetext{
${ }^{1}$ In case the MR has fewer than $\mathrm{N}$ transceivers, e.g.; $L$, it can apply the protocol below to $L$ channels at a time.
}

In the proposed scheme, to assign valid IDs for $M$ SUs, the required IDs' field length is $M+1$ bits such that the ID's bits corresponding to $S U_{i}$ are all set to zeros except bit number i. For instance, for $S U_{1}$ the ID is "0000010" if $M=6$ and for $S U_{2}$ the ID is "0100" where $M=3$. Table II shows an example of selected IDs when $M=5$. It is worth noting that in one cluster of WMNs the number of SUs usually is not large, and only a short ID field is required. The reason for this choice of ID will become clear when we discuss our strategy.

We propose at SUs in a CR-WMN cluster adopt one of the following two modulation schemes, either $P N C_{2}$ or $P N C_{1}$ (which are explained in detail in subsections IV-A and IV-B, respectively), in the network operation to transmit their IDs concurrently where the ID coding proposed earlier is used in both scenarios. The received signals are decoded to extract the stations' IDs, or to detect collisions efficiently. Each scenario has its advantages and disadvantages in terms of $p_{s}$ for transmission and network setup overhead as illustrated in Section VI, as well as simplicity.

\section{A. $\mathrm{PNC}_{2}$ (PNC with two Modulation Schemes):}

Let there be 2 transmitters (SUs), say $A$ and $B$, and one receiver (MR) where the SUs send their IDs to the MR. $A$ and $B$ use one channel at the same time to transmit requests for uplink channels to the MR. A transmitter can employ standard Binary phase-shift keying (BPSK) modulation, call it $M_{1}$, in which the symbol can be either $A_{1}$ or $A_{0}$, while the other transmitter can employ the antipodal to the standard BPSK, call it $M_{2}$ which corresponds to $B_{1}$ and $B_{0}$ as shown in Figure 1. In this scheme, the two modulation schemes, $M 1$ and $M 2$ are used, and an SU either uses $M 1$ or $M 2$ as its modulation scheme. This is sufficient to decode the received signals as the combinations of the bits are uniquely decodable, as shown in Table I and Figure 1. Signals transmitted by $A$ and $B$ SUs are shown in dashed lines in Figure 1. In this case, these two transmitters can be regarded as transmitting orthogonally to each other. The BER is similar to orthogonal QPSK if both $A$ and $B$ transmit simultaneously. The BPSK signal with antipodal signalling is used by the MR to decode the signals from the SUs transmitters, $A$ and $B$, by using the resultant phases and the energy of the received signals as shown in Table I.

Definition 4.1: $\mathrm{PNC}_{2}$ collision $\left(\mathrm{PNC}_{2 c}\right)$ : the collision on a channel is due to either a transmission where more than 2 SUs access the channel simultaneously or 2 SUs access the channel simultaneously, but both have selected the same modulation technique because SUs behave independently.

To detect $P N C_{2 c}$ in this scenario, assume some SUs use modulation technique $M_{1}$ and the others use modulation technique $M_{2}$. The phase shifts for the received signals are used to detect collisions. If the phase shifts do not match the values in Table I, and under noise free operation conditions, then this indicates a collision. When more than two SUs use the same channel, then none of the detected phase shifts will correspond to $A_{1} B_{0}$ nor $A_{0} B_{1}$ (due to using the proposed 
SUs IDs selection method as in Table II). Also, some received bit signals will have no energy. Thus, in $\mathrm{PNC}_{2}$ decoding the received signals mainly depends on their received phase shifts.

When both SUs transmit either '0's or '1's bits and their modulation technique is the same, e.g.; $M_{1}$, then the received signals' phase shifts are $\pi$ or 0 , respectively. For instance, if $S U_{1}$ transmits "11" and $S U_{2}$ transmits "11", the received bits at MR are "11" as well with a phase shift equals 0 . However, corruption happens when one of the transmitters transmits ' 1 ' bit and the other transmits ' 0 ' bit when both SU transmitters have the same transmission energy. In this case, the received signal can not be used for decoding because its energy is zero. We call this signal ' $\mathrm{X}$ '. However, this case is considered in the other scheme, $P N C_{1}$, to decode the received signal for a pair of SU transmitters.

\section{B. $P N C_{1}$ (PNC with One Modulation Scheme):}

In this scheme, all SUs use one modulation scheme either $M 1$ or $M 2$, and the MR can indicate which modulation scheme can be used. Thus, the decoding process shown in Table I does not work. When any pair of SUs transmit their IDs using the same BPSK modulation scheme $\left(M_{1}\right.$ or $\left.M_{2}\right)$, the bits of the IDs are XORed using $P N C$ (PXOR), which is shown in Table III. The SUs' IDs must be chosen such that: (a) PXOR of any pair of SUs IDs is unique, and (b) PXOR of more than two SUs IDs is distinguishable from PXOR of two SUs IDs.

However, when the SUs' IDs are selected as indicated above, then they satisfy the above two conditions ( $a$ and $b$ ), and the received signals can be decoded correctly. For example, if $M=2$ the IDs for $S U_{0}$ and $S U_{1}$ are "001" and "010", respectively, and their received code word at MR is "OXX" if both SUs use the same channel which can be decoded uniquely, since each ID has exactly one bit equal to ' 1 '. It should be noted that when $S U_{i}$ and $S U_{j}$ transmit their IDs using the same modulation scheme, bits $i$ and $j$ will both have no energy (X), and the rest of the bits will be zeroes. Notice that if only $M$ bits are used for the ID packet, the IDs will be "01" and "10" for $S U_{0}$ and $S U_{1}$, respectively. Thus, if those SUs use the same channel, the received code word at MR is " $\mathrm{XX}$ " which contains no energy and will not be detected. Therefore, the extra ' 0 ' bit to the left is necessary to detect the SUs transmissions at the MR.

Definition 4.2: $P N C_{1}$ collision $\left(P N C_{1 c}\right)$ : A transmission on a channel where more than 2 SUs have accessed the channel simultaneously.

If $P N C_{1 c}$ occurs, the received signals values for $k$ bits are all '0's with the same energy level (given the $k$ SUs received energy levels are the same, $k \geq 3)$. The rest of $(M-k+1)$ bits are ' 0 's and their energies are equal and have a higher energy level than the $k$ bits. Thus, no ' $\mathrm{X}$ ' bits are received. Hereby, the collision is detectable by the MR because the received code word bits are all zeros with $\pi$ as their phase shifts (if $M_{1}$ is employed by SUs). Thus, decoding the received signals mainly depends on their received power levels.

\section{COMmunication Protocol}

The communication and control signals protocol is explained in this section. Mainly, we are interested in setup time reduction in CR-WMNs using $P N C$. Initially, the SUs adopt either $P N C_{1 o r 2}$ as their modulation technique through its operation. The Communication Protocol for $P N C_{1}$ or $P N C_{2}$ :

Whenever channels availability at MR changes, the MR advertises its available $\mathrm{N}$ channels again to SUs by broadcasting the available channel numbers information over all $\mathrm{N}$ channels. If an $\mathrm{SU}$ was tuned to a channel which is no longer available, this SU waits for a while if no packets are received from MR or overheard. Then, the SU starts scanning the spectrum until it finds an active available channel, and tunes its transceiver to the channel. Figure 2 shows a time line for exchanging the control signals and executing the communication protocol between MR and two SUs in one time slot, assuming the SUs transmissions are perfectly synchronized and the environment is noise free. However, a channel may be used by one SU where $P N C$ is not performed. The communication protocol steps are as follows:

1) The MR sends a pull signal over all its $N$ channels, which also includes the list of all free channels. Each SU therefore becomes aware of all free channels.

2) When an SU receives the pull signal, the $S U$ selects one channel randomly out of $\mathrm{N}$ and sends a channel request packet containing its ID to the MR on that channel. Transmissions need to be synchronized for $P N C$ implementation.

3) The MR receives a set of channels requests by SUs over some/all of its channels. At this stage, the MR decides the received packet over a channel is due to either 1 or $2 \mathrm{SU}$ transmissions (no collision) or more than two SUs transmissions (collision).

4) The MR processes the successfully received channel requests and assigns channels to SUs. Then, the MR broadcasts the channel allocation over each channel on which a request was received. For instance, if two SUs requests are received, the MR broadcasts one packet which contains the channel assignment information for both SUs. But, when a collision occurs, the MR does not broadcast channel assignment packet, thus the SUs (where $|\mathrm{SUs}| \geq 3$ ) will try to send their channel requests in the next time slot and on a channel which is selected randomly from among the remaining channels.

5) The SUs who sent requests in step 2, stay tuned to the same channel to receive their channel allocation packet sent by their MR. At this stage, there are two possibilities: 1st, the SU does not receive a reply from the MR due to either packets' collision or there are no more available channels. 2nd, the SU receives channels allocation packet.

6) Each SU which receives the channel allocation packet sends a positive (+) ACK signal to the MR, containing its ID, over the channel which was selected in step 2. The MR uses the proposed $P N C$ model to decode the 


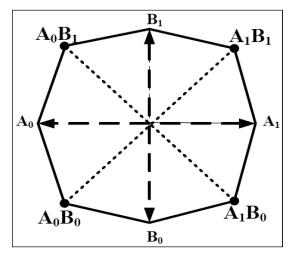

Fig. 1. Constellation diagram for two transmitters $A$ and $B$.

TABLE I

SIGNALS CODING AND DECODING FOR TWO TRANSMITTER NODES AND ONE RECEIVER NODE IN $\mathrm{PNC}_{2}$ SCHEME.

\begin{tabular}{|c|c|c|c|}
\hline$A$ & $B$ & PNC Signal & Decoded Signals \\
\hline 0 & 0 & signal with the phase difference $e^{j\left(-\frac{3 \pi}{4}\right)}$ & $A_{0}, B_{0}$ \\
\hline 0 & 1 & signal with the phase difference $e^{j\left(\frac{3 \pi}{4}\right)}$ & $A_{0}, B_{1}$ \\
\hline 1 & 0 & signal with the phase difference $e^{j\left(-\frac{\pi}{4}\right)}$ & $A_{1}, B_{0}$ \\
\hline 1 & 1 & signal with the phase difference $e^{j\left(\frac{\pi}{4}\right)}$ & $A_{1}, B_{1}$ \\
\hline
\end{tabular}

received signal. In case both SUs send +ACK signals, their transmission must also be synchronized.

7) Steps 1 through 6 are repeated until either all channels are assigned at the MR, if the number of SUs is greater than N. Or, all SUs are assigned channels, if $|\mathrm{SUs}|<$ $\mathrm{N}$.

8) The MR broadcasts a signal over the available channels to inform the SUs to start their uplink data transmissions where these data packets will be relayed by the MR to the gateway of the CR-WMN.

\section{RESUlTS AND DISCUSSION}

In the section, four different schemes are compared using simulation. The 1st scheme is the classical scheme where one channel is used as a CCC for channel allocation to SUs by MR in the WMN cluster; call it (one CCC). The SUs' access channel protocol is CSMA/CA with the RTS/CTS used in IEEE 802.11 networks. We used OPNET simulation tool to implement the one CCC scheme.

The 2nd scheme is time slotted where neither $P N C_{1}$ nor $P N C_{2}$ is used, call it (No $P N C$, random), for a request to be successful, it must be the only one on the channel. The 3rd scheme is time slotted with $P N C_{1}$, call it $\left(P N C_{1}\right.$, random). The 4th scheme is time slotted with $P N C_{2}$, call it $\left(P N C_{2}\right.$, random). In the 2nd, 3rd, and 4th schemes time is slotted. At the beginning of each time slot, all SUs which have not been assigned channels yet, compete for the $\mathrm{N}$ channels such that each SU selects randomly a channel to send a channel Request (Req) packet to the MR without coordination among them. In these three schemes there are no RTS/CTS handshakes. Thus, the collision event happens only at the beginning of the time slot as shown in Figure 2. In the 2nd scheme, if more than one SU accesses a channel, a collision occurs. However, in the 3rd and 4th schemes, if more than 2 SUs access a channel, a collision occurs.

When a collision occurs on a channel the MR will not be able to decode the received signals, SUs' IDs, correctly. Subsequently, those SUs will try in the following time slots until channels are assigned to them by the MR (if there are still available channels). We assume that the communication

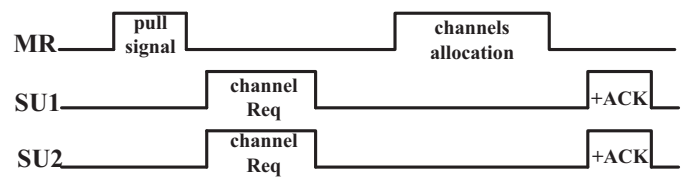

Fig. 2. Control Signals for one time slot in $P N C$.
TABLE II THE SET OF VALID IDS FOR FIVE SUS IN A WMN CLUSTER.

\begin{tabular}{|c|c|}
\hline SU & SU's ID \\
\hline 0 & 000001 \\
\hline 1 & 000010 \\
\hline 2 & 000100 \\
\hline 3 & 001000 \\
\hline 4 & 010000 \\
\hline
\end{tabular}

TABLE III

THE XOR OPERATION IN $P N C_{1}$ SCHEME.

\begin{tabular}{|c|c|c|}
\hline$A$ & $B$ & PXOR \\
\hline 0 & 0 & 0 \\
\hline 0 & 1 & $\mathrm{X}$ \\
\hline 1 & 0 & $\mathrm{X}$ \\
\hline 1 & 1 & 1 \\
\hline
\end{tabular}

is perfectly synchronized and the environment is noise free. For simplicity, we assume that the propagation and processing delays are included in a time slot which is shown in Figure 2. To measure the overall delay for the time slot, we used OPNET simulation tool.

\section{A. Probability of Success:}

Figure 3 shows the $p_{s}$ for the 2 nd scheme where $P N C_{1}$ is not used, call it (No $P N C_{1}$, random), and the 3rd scheme $\left(P N C_{1}\right.$, random) when the number of channels, $\mathrm{N}$, is $4,6,8$ and 10, respectively, and for different numbers of SUs. Also, Figure 4 shows the $p_{s}$ for the 2nd scheme when $\mathrm{PNC}_{2}$ is not used, call it (No $P N C_{2}$, random), and the 4th scheme $\left(\mathrm{PNC}_{2}\right.$, random) when the number of channels, $N$, is $4,6,8$ and 10 , respectively, and for different numbers of SUs. $P_{s}$ is the probability that an SU successfully transmits a channel request packet and is therefore assigned a data channel. Clearly, the results show that using either $P N C_{1}$ or $P N C_{2}$ schemes increases the $p_{s}$ for channel requests packets transmissions which decreases the channel assignment time delay, as will be shown next. Figures 3 and 4 show $P N C_{1}$ scheme outperforms $P N C_{2}$ in terms of $p_{s}$ for different $N$ and $M$ when it is employed by SUs.

\section{B. Setup time overhead:}

Figures 5, 6, 7 and 8 show the setup time overhead for the four schemes explained earlier for different numbers of available channels at MR and different numbers of SUs in a CR-WMN. Clearly, our proposed scheme which uses $P N C$ $\left(P N C_{1}\right.$ or $\left.P N C_{2}\right)$ outperforms both the 1st and the 2nd schemes, the one CCC and the time slotted without PNC schemes, respectively. Table IV shows the setup time overhead reduction percentages for the 3rd and the 4th schemes compared to the 1st scheme for a sample of different $N$ and $M$. Also, $P N C_{1}$ scheme outperforms $P N C_{2}$ scheme, because the setup time overhead is less when $P N C_{1}$ scheme is employed by SUs rather than $P N C_{2}$. Also, Table IV shows the reduction in setup time overhead for $P N C_{1}$ scheme is higher than $P N C_{2}$ for a sample of different $N$ and $M$. An interesting observation is that the overhead increase almost linearly if 

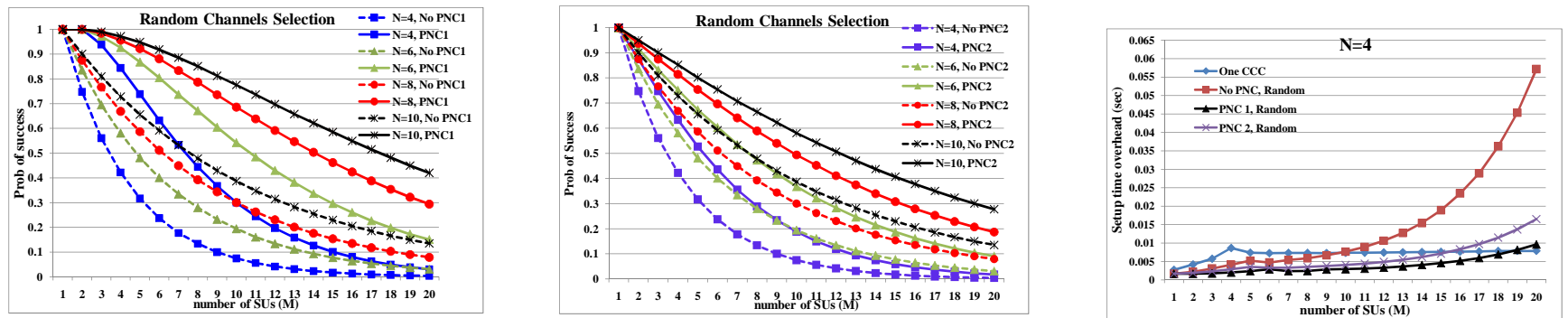

Fig. 3. $P N C_{1}$ prob of success with random chan- Fig. 4. $P N C_{2}$ prob of success with random chan- Fig. 5. CR network setup time overhead if N=4. nels selection where $\mathrm{N}$ is $4,6,8$ and 10 , respectively. nels selection where $\mathrm{N}$ is $4,6,8$ and 10 , respectively.

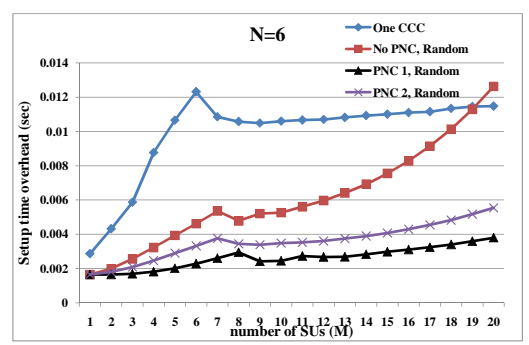

Fig. 6. $\quad$ CR network setup time overhead if $\mathrm{N}=6$

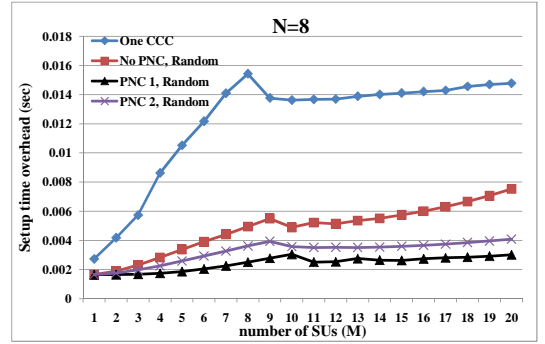

Fig. 7. $\quad$ R network setup time overhead if $\mathrm{N}=8$.

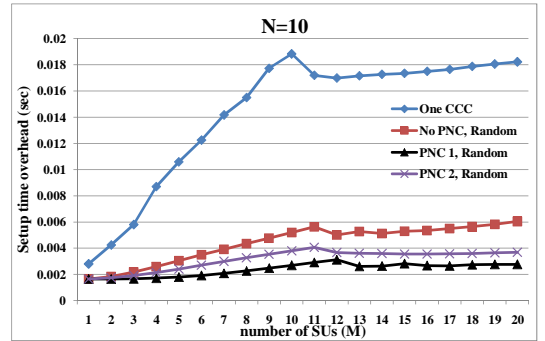

Fig. 8. CR network setup time overhead if $\mathrm{N}=10$.
TABLE IV

SETUP TIME SAMPLE OVERHEAD REDUCTION PERCENTAGES FOR THE THIRD AND FOURTH SCHEMES COMPARED TO THE FIRST SCHEME.

\begin{tabular}{|c|c|c|c|c|}
\hline Scheme & $\mathrm{N}=4, \mathrm{M}=6$ & $\mathrm{~N}=4, \mathrm{M}=10$ & $\mathrm{~N}=10, \mathrm{M}=6$ & $\mathrm{~N}=10, \mathrm{M}=10$ \\
\hline$P N C_{1}$ & $60 \%$ & $60 \%$ & $84 \%$ & $85 \%$ \\
\hline$P N C_{2}$ & $54 \%$ & $45 \%$ & $77 \%$ & $79 \%$ \\
\hline
\end{tabular}

$|\mathrm{SUs}| \leq N$. However, if $|\mathrm{SUs}|>N$, then the overhead starts to decrease before increasing again. Actually, it consistently peaks at $\mathrm{N}, \mathrm{N}+1, \mathrm{~N}+1$ and $\mathrm{N}+2$ for one CCC, (No $P N C$, random $),\left(P N C_{2}\right.$, random $)$ and $\left(P N C_{1}\right.$, random) schemes, respectively.

\section{CONCLUSIONS}

In this paper, we propose a new scheme for channel request and assignment in CR-WMNs. Our scheme is based on using $P N C$ where up to 2 SUs may select the same channel to send channel request packets to the MR. The proposed $P N C$ schemes are $P N C_{1}$ and $P N C_{2}$ where decoding the received signals at MR depends on their received energy and phases shifts, respectively. Simulation results show that our scheme outperforms the one CCC scheme and the scheme in which time is slotted and an SU selects randomly one channel out of $\mathrm{N}$ channels to send the channel request packet to MR without using $P N C$. The new scheme has much lower setup time overhead which is required to allocate $\mathrm{N}$ channels to the set of SUs in the cluster. Also, the probability of success $\left(p_{s}\right)$ for packet delivery by SUs is higher when $P N C$ is employed in the slotted time scheme. Also, the simulation results show $P N C_{1}$ scheme outperforms $P N C_{2}$ scheme in terms of $p_{s}$ and setup time overhead if it is employed by SUs. In our future work, we will evaluate the BER under multipath transmissions in $\mathrm{PNC}_{2}$.

\section{REFERENCES}

[1] I. F. Akyildiz, W.-Y. Lee, M. C. Vuran, and S. Mohanty, "Next generation/dynamic spectrum access/cognitive radio wireless networks: a survey". Comput. Netw., vol. 50, no. 13, pp. 2127-2159, 2006.

[2] I. Mitola, J., "Cognitive radio for flexible mobile multimedia communications". In IEEE MoMuC 1999 International Workshop, pp.3-10.

[3] I. Mitola, J., "Cognitive radio: An integrated agent architecture for software defined radio". PhD Thesis, KTH Royal Institute of Technology, 2000.

[4] A. Ghasemi and E. Sousa, "Collaborative spectrum sensing for opportunistic access in fading environments". In IEEE DySPAN 2005.

[5] X. Chen, Z.-S. Bie, and W.-L. WU., "Detection efficiency of cooperative spectrum sensing in cognitive radio network". The Journal of China Universities of Posts and Telecommunications, vol. 15, pp. 1-7, 2008.

[6] A. Chia-Chun Hsu, D. Weit, and C.-C. Kuo, "A cognitive mac protocol using statistical channel allocation for wireless ad-hoc networks". IEEE WCNC 2007, pp. 105-110.

[7] Q. Zhao, L. Tong, A. Swami, and Y. Chen, "Decentralized cognitive mac for opportunistic spectrum access in ad hoc networks: A pomdp framework". IEEE JSAC vol. 25, pp. 589-600, 2007.

[8] J. Zhao, H. Zheng, and G.-H. Yang, "Distributed coordination in dynamic spectrum allocation networks". In IEEE DySPAN 2005.

[9] R. Ahlswede, N. Cai, S.-Y. Li, and R. Yeung, "Network information flow". IEEE Transactions on Information Theory, vol. 46, pp. 12041216, Jul 2000.

[10] Y. Kondareddy and P. Agrawal, "Synchronized MAC Protocol For Multihop Cognitive Radio Networks". In IEEE ICC 2008.

[11] C. Doerr, D. Sicker, D. Grunwald, "Dynamic Control Channel Assignment in Cognitive Radio Networks using Swarm Intelligence". In IEEE GLOBECOM 2008

[12] S. Zhang, S. Liew, and P. Lam, ”Hot Topic: Physical-Layer Network Coding". In MobiCom 2006.

[13] N. Baldo, A. Asterjadhi, and M. Zorzi, "Multi-channel medium access using a virtual network coded control channel," In IWCMC 2009.

[14] Yung-Szu Tu and Gregory J. Pottie, "Coherent cooperative transmission from multiple adjacent antennas to a distant stationary antenna through AWGN channels". In the 55th IEEE VTC 2002.

[15] R. Mudumbai, G. Barriac, and U. Madhow, "On the Feasibility of Distributed Beamforming in Wireless Networks". IEEE TRANSACTIONS ON WIRELESS COMMUNICATIONS, VOL. 6, NO. 4, APRIL 2007.

[16] M. Simon, E. Valles, C. Jones, R. Wesel and J. Villasenor, "InformationReduced Carrier Synchronization of BPSK and QPSK Using Soft Decision Feedback". 44th Annual Allerton Conference Allerton House, 2006. 